OPEN ACCESS

Edited by:

Jesus L. Romalde,

Universidade de Santiago

de Compostela, Spain

Reviewed by:

Catherine Eichwald,

University of Zurich, Switzerland

Majid Fasihi Harandi,

Kerman University of Medical

Sciences, Iran

*Correspondence:

Wenbao Zhang

wenbaozhang2013@163.com

Jun Li

1742712944@163.com,

1742712944@qq.com

Specialty section:

This article was submitted to

Evolutionary and Genomic

Microbiology,

a section of the journal

Frontiers in Microbiology

Received: 16 December 2017

Accepted: 12 November 2018

Published: 29 November 2018

Citation:

Bao J, Zheng $H$, Wang $Y$ Zheng X, He L, Qi W, Wang T, Guo B, Guo G, Zhang Z, Zhang W, Li J and McManus DP (2018) Echinococcus granulosus Infection Results in an

Increase in Eisenbergiella and Parabacteroides Genera in the Gut of Mice.

Front. Microbiol. 9:2890. doi: 10.3389/fmicb.2018.02890

\section{Echinococcus granulosus Infection Results in an Increase in Eisenbergiella and Parabacteroides Genera in the Gut of Mice}

\author{
Jianling Bao ${ }^{1,2}$, Huajun Zheng ${ }^{3,4}$, Yuezhu Wang ${ }^{3}$, Xueting Zheng ${ }^{1}$, Li He ${ }^{1}$, Wenjing Qi ${ }^{1}$ \\ Tian Wang ${ }^{1}$, Baoping Guo', Gang Guo ${ }^{1}$, Zhaoxia Zhang ${ }^{1}$, Wenbao Zhang ${ }^{1,2 *}$, Jun Li ${ }^{1 *}$ \\ and Donald P. McManus ${ }^{5}$
}

${ }^{1}$ State Key Laboratory of Pathogenesis, Prevention and Treatment of High Incidence Diseases in Central Asian, The First Affiliated Hospital of Xinjiang Medical University, Urumai, China, ${ }^{2}$ College of Public Health, Xinjiang Medical University, Urumqi, China, ${ }^{3}$ Key Laboratory of Reproduction Regulation of NPFPC, SIPPR, IRD, Fudan University, Shanghai, China, ${ }^{4}$ Shanghai-MOST Key Laboratory of Health and Disease Genomics, Chinese National Human Genome Center at Shanghai, Shanghai, China, ${ }^{5}$ Molecular Parasitology Laboratory, QIMR Berghofer Medical Research Institute, Brisbane, QLD, Australia

Cystic echinococcosis (CE) is a chronic infectious disease caused by Echinococcus granulosus. To confirm whether the infection impacts on the gut microbiota, we established a mouse model of $E$. granulosus infection in this study whereby BALB/c mice were infected with micro-cysts of $E$. granulosus. After 4 months of infection, fecal samples were collected for high-throughput sequencing of the hypervariable regions of the 165 rRNA gene. Sequence analysis revealed a total of 13,353 operational taxonomic units (OTUs) with only $40.6 \%$ of the OTUs having genera reference information and 101 of the OTUs were significantly increased in infected mice. Bioinformatics analysis showed that the common core microbiota were not significantly changed at family level. However, two genera (Eisenbergiella and Parabacteroides) were enriched in the infected mice $\left(P_{\text {AMOVA }}<0.05\right)$ at genus level. Functional analysis indicated that seven pathways were altered in the E. granulosus Infection Group compared with the Uninfected Group. Spearman correlation analysis showed strong correlations of IgG, IgG1 and IgG2a with nine major genera. E. granulosus cyst infection may change the gut microbiota which may be associated with metabolic pathways.

Keywords: Echinococcus granulosus, cystic echinococcosis, mice, microbiome, immunoglobins

\section{INTRODUCTION}

Cystic echinococcosis (CE) is a cosmopolitan zoonosis caused by the cystic stage of the dog tapeworm Echinococcus granulosus (McManus et al., 2012). The disease causes serious health problems and economic losses, especially in Central Asia (including western China), northern Africa and South America (McManus et al., 2012). E. granulosus requires two hosts [an intermediate host including sheep, goats, cattle or wild herbivores and a definitive host such as dogs (or wolves and other carnivores)] to complete its life-cycle. Humans also become infected as an incidental host by ingesting eggs released from E. granulosus in carnivore feces. After hatching in the stomach and 
intestine, the eggs (oncospheres) penetrate the gut mucosa, enter the blood circulation and finally develop in internal organs mainly the liver (70\%) and lungs (20\%) of humans. Serological studies showed that in endemic areas, $4-26 \%$ of the population were seropositive against E. granulosus antigens (Gavidia et al., 2008), indicating that a large number of individuals had been infected. CE infection strongly impacts on host immune responses (Zhang et al., 2003, 2012) with high levels of antibodies, particularly IgG1, IgG4 and IgE (Zhang et al., 2003) and predominant Th2 cytokines including IL-4, IL-5, IL-6, and IL-10 (Rigano et al., 1997; Casaravilla et al., 2014), indicating that the host immune response against CE differs from a bacterial or viral infection (Zeng et al., 2017).

The gut microbiota play an important role in human health (Chen et al., 2017) impacting on metabolism, immunity, development and the behavior of the host (Thaiss et al., 2016). In addition, microbiota components are impacted by medical conditions such as cancer (Jensen et al., 2015; O'Keefe, 2016; Sonnenburg and Backhed, 2016). Similar changes occur in experimental models as well (Gkouskou et al., 2014; Yu et al., 2018). Studies showed that helminth infection in the gut induced typical Th2 immune responses which may control the mcriobiota in the gut of mice (Ramanan et al., 2016; Guernier et al., 2017; Wegener Parfrey et al., 2017). However, it is not known whether E. granulosus infection impacts on the gut microbiota of humans or mice. Mice have been used for E. granulosus larval infection including primary (Zhang et al., 2001) and secondary infection (Gottstein, 2001; Mourglia-Ettlin et al., 2016). Mice models play an important role in studies of developmental biology and host specificity in echinococcosis (Nakaya et al., 2006). Recently, mouse models were successfully used for drug screening and development (Elissondo et al., 2007; Wang et al., 2017). To increase the success of secondary infection, we developed a method using micro-cysts cultured in vitro to infect mice (Zhang et al., 2005), and obtained more than $70 \%$ of cyst recovery from 50 PSC-generated cysts.

In this study, BALB/C mice were infected with microcysts of E. granulosus and their fecal samples were collected for sequencing the variable regions of $16 \mathrm{~S}$ rRNA genes of gut commensal bacteria to determine their composition and diversity. We show that E. granulosus impacted on the gut microbiota of the mice with microbiota changes likely being associated with the altered host immune status in infected individuals.

\section{MATERIALS AND METHODS}

\section{Ethics Statement}

The protocols for using mice in the study were approved by the Ethics Committee of The First Affiliated Hospital of Xinjiang Medical University (FAH-XMU, Approval No. IACUC20120625003). The "Guidelines for the Care of Laboratory Animals" by the Ministry of Science and Technology of the People's Republic of China (2006) were rigidly followed in the use of these animals.

\section{Preparation of Cultured E. granulosus Hydatid Cysts}

Fresh E. granulosus sensu stricto protoscoleces (PSC) were aspirated from hydatid cysts from sheep livers collected from a slaughterhouse in Urumqi, Xinjiang Uyghur Autonomous Region, China. The PSC were digested with $1 \%(\mathrm{w} / \mathrm{v})$ pepsin and cultured to obtain echinococcal cysts using published procedures (Zhang et al., 2005; Wang et al., 2014).

\section{Animal Infection and Sample Collection}

Pathogen-free female BALB/c mice, aged 6 weeks, were purchased from Beijing Vital River Laboratory Animal Technology Co., Ltd. All animals were housed at the animal facility of the FAH$\mathrm{XMU}$. The BALB/c mice were randomly divided into two groups: E. granulosus infected group (Infected Group) and uninfected group (Uninfected Group). The mice in the E. granulosus Infection Group were intraperitoneally (i.p) transplanted with 35 small (diameter, 200-300 $\mu \mathrm{m}$ ) hydatid cysts suspended in $0.4 \mathrm{~mL}$ RPMI 1640 medium through a $1.0 \mathrm{~mL}$ syringe as described in our previous study (Zhang et al., 2005; Wang et al., 2016). Every mouse in the Uninfected Group was i.p. injected with $1.0 \mathrm{~mL}$ RPMI 1640 medium. After 4 months, the stool of each mouse was collected daily and about $1 \mathrm{~g}$ stool was collected over 1 week. The stool samples were stored at $-80^{\circ} \mathrm{C}$ until use.

\section{Antibody Isotype and Subtype Assays}

Mice were sacrificed after the last fecal sample collection, then serum samples were obtained from peripheral blood and stored at $-20^{\circ} \mathrm{C}$ for use. The sera were analyzed by ELISA for different immunoglobulins to hydatid cyst fluid (HCF) including IgG, IgG1, IgG2a, IgG2b and IgG3 and IgM. In brief, each well of MaxiSorb immune-plates (Nunc International) was coated with $100 \mu \mathrm{L}$ of sheep HCF antigens at a concentration of $2 \mathrm{mg} / \mathrm{mL}$ in carbonate/bicarbonate buffer (pH 9.6) (Kittelberger et al., 2002) and incubated overnight at $4^{\circ} \mathrm{C}$. After three washes with PBS, the wells were each blocked with $200 \mu \mathrm{L}$ of $5 \%$ (v/v) skim milk in PBS for $1 \mathrm{~h}$ at $37^{\circ} \mathrm{C}$. Mouse serum was diluted 1-200. For each well, $100 \mu \mathrm{L}$ of the diluted serum was added and incubated at $37^{\circ} \mathrm{C}$ for $1 \mathrm{~h}$. After three washes with PBS containing $0.05 \%$ Tween 20 (PBST), each well was added $100 \mu \mathrm{L}$ of diluted anti-mouse monoclonal IgG, IgG1, IgG2a, IgG2b and IgG3 and IgM conjugate (BETHYL). The reaction was developed by adding $100 \mu \mathrm{L}$ of 2,2-azino-di-[ethyl-benzothiazoline sulfonate] substrate solution (Sigma). After incubation for $30 \mathrm{~min}$ in the dark at room temperature, optical density values were read at $405 \mathrm{~nm}$ by an ELISA reader (Thermo, Waltham, United States).

\section{Echinococcal Cyst Number and Size}

The abdominal cavity of each mouse was opened after sacrifice, and hydatid cysts in the cavity were removed and the numbers of hydatid cysts were counted and their sizes were measured.

\section{PCR Amplification and 16S rRNA Gene Sequencing}

Genomic DNA was extracted from stool samples using KAPA HiFi HotStart ReadyMix Kits (Kapa Biosciences, Woburn, MA, 
TABLE 1 | The number and average size of cysts in the E. granulosus infection group.

\begin{tabular}{|c|c|c|c|c|c|c|c|c|c|c|c|c|c|c|}
\hline & Eg1 & Eg2 & Eg3 & Eg4 & Eg5 & Eg11 & Eg12 & Eg13 & Eg14 & Eg15 & Eg31 & Eg32 & Eg33 & Eg35 \\
\hline Number of cysts & 12 & 15 & 17 & 37 & 21 & 16 & 17 & 14 & 15 & 11 & 12 & 8 & 23 & 13 \\
\hline Average size of cysts(cm) & 0.58 & 0.54 & 0.58 & 0.72 & 0.74 & 0.55 & 0.67 & 0.42 & 0.49 & 0.71 & 0.55 & 0.75 & 1.01 & 0.77 \\
\hline
\end{tabular}

Eg, mice infected with E. granulosus.
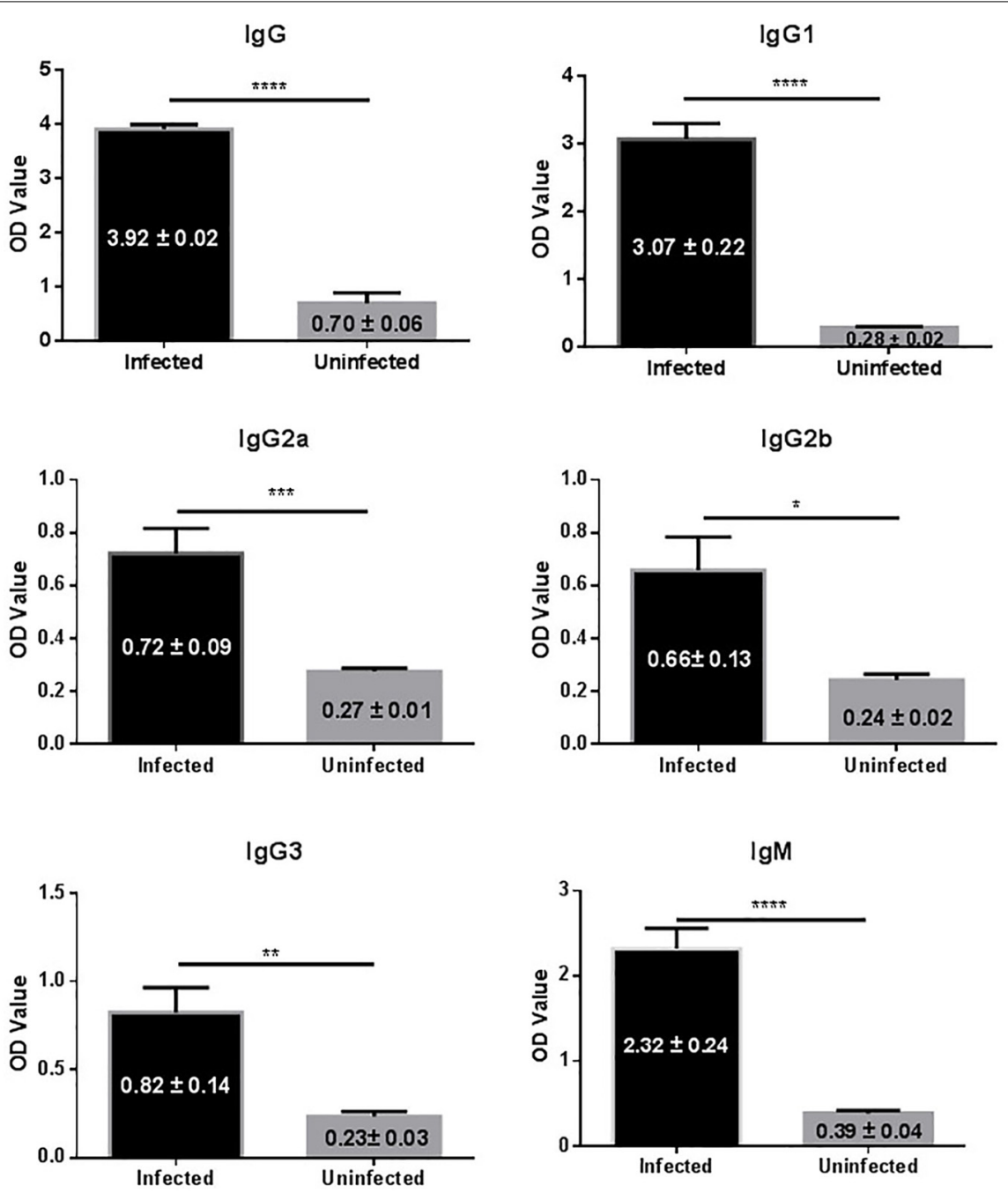

FIGURE 1 | lgG antibody isotypes and subtypes in sera of mice infected with E. granulosus. ${ }^{*} p<0.05,{ }^{* *} p<0.01,{ }^{* * *} p<0.005,{ }^{* * * *} p<0.001$.

United States). The V3-V4 hypervariable region of the 16S rRNA gene was amplified by PCR and sequenced; the length of the V3V4 hypervariable region was approximately 469 bp. Amplicon pools were prepared for sequencing with AMPure XT beads (Beckman Coulter Genomics, Danvers, MA, United States) and quantification with the Library Quantification Kit for Illumina (Kapa Biosciences, Woburn, MA, United States), respectively. The libraries were sequenced on 300PE MiSeq runs.

\section{Bioinformatics and Statistical Analysis}

Mothur (version 1.39.5) was used to assemble the paired FASTQ files (Schloss et al., 2011). The selected quality DNA sequences were confirmed using the following criteria: (1) no contaminant sequences, (2) containing no ambiguous bases, (3) the size length $\geq 350 \mathrm{bp}$, (4) containing no chimeric sequences, and (5) primers were trimmed. The average length of selected DNA sequences was $414 \mathrm{bp}$ (350-446 bp). The selected DNA sequences were then grouped into operational taxonomic units (OTUs) by comparing with SILVA reference databases (V128) (Quast et al., 2013) at $97 \%$ similarity. The minimum reads number of samples $(24,097)$ was used for data normalization. Community richness, evenness and diversity analysis (Shannon, Simpson, Shannoneven, Simpsonenven, ACE, Chao and Good's coverage) were analyzed using the Mothur T-test (with 95\% confidence 
TABLE 2 | The diversity evaluation of the microbiomes of mice infected with cystic echinococcosis and uninfected mice.

\begin{tabular}{|c|c|c|c|c|c|c|c|c|c|}
\hline \multirow[b]{2}{*}{ Group } & \multirow[b]{2}{*}{ Sample } & \multirow[b]{2}{*}{ OTUs } & \multirow[b]{2}{*}{ Coverage (\%) } & \multicolumn{2}{|c|}{ Richness } & \multicolumn{2}{|c|}{ Evenness } & \multicolumn{2}{|c|}{ Diversity } \\
\hline & & & & Chao & ACE & Simpsoneven & Shannoneven & Shannon & Simpson \\
\hline Infected & 14 & 9,118 & 98.50 & $18,212.718$ & $26,873.315$ & 0.01444 & 0.646977 & 5.899138 & 0.007595 \\
\hline Uninfected & 11 & 8,423 & 98.25 & $16,846.368$ & $25,099.202$ & 0.01675 & 0.660714 & 5.972012 & 0.007084 \\
\hline
\end{tabular}

Infected, mice infected with E. granulosus; Uninfected, uninfected mice; OTUs, operational taxonomic units; Chao, Chao index, ACE, ACE index.

intervals, $p$-value $<0.05)$. Taxonomy was assigned using the online software Ribosomal Database Project (RDP) classifier (80\% threshold) (Wang et al., 2007) based on the RDP (Cole et al., 2009). LEfSe (Segata et al., 2011) was also performed to detect abundance taxa ( $p$-value $<0.05$ ) between the two groups and estimate linear discriminant analysis effect size (LDA score >2.0). Then Mothur was performed to check the LEfSe results using "metastats" command. Differences among the two groups were also assessed using Analysis of Molecular Variance (AMOVA) in Mothur. Microbiome functions were analyzed using PICRUSt (Langille et al., 2013) based on the KEGG pathways by normalizing the $16 \mathrm{~S}$ rRNA copy numbers. The input file (biom file) of PICRUSt was calculated using the Mothur software command "classify.otu" and "make.biom", and then the input file was uploaded to the online PICRUSt for function analysis. Differences were determined using STAMP (Parks et al., 2014).

\section{Correlation of Antibody Isotypes and Bacteria}

Statistical analysis program-R Package was performed to calculate the coefficient relationship between bacterial genera present and immunoglobulin isotypes using the non-parametric Spearman rank correlation algorithm. A coefficient of $>0.68$ or $<-0.68$ was considered to represent strong correlation (Taylor, 1990).

\section{RESULTS}

\section{Infection and Blood Serum Isotypes}

In this study 14 mice were transplanted with 35 micro-cysts of E. granulosus. All the mice were successfully infected with an average number of $16(\mathrm{SD} \pm 7.0)$ cysts and an average size of $6.4 \mathrm{~mm}(0.1-21 \mathrm{~mm})$ in diameter (Table 1). Serological antibody tests showed that these infected mice had a predominantly IgG1 antibody response against HCF antigens, followed by IgG2b, IgG2a and IgG3 (Figure 1), indicating E. granulosus infection induced a predominant Th2 response.

\section{Bacterial Populations in the Stool Samples}

Stool samples from the 25 mice were collected for gastrointestinal microbiota analysis, including 14 samples from the mice infected with cysts of E. granulosus (Infected Group) and 11 control samples from mice without infection (Uninfected Group,). A total of 1,383,569 16S rRNA genes were identified by highthroughput DNA sequencing analysis after filtering through quality control filters. The gene numbers ranged from 24,097 (from one in Uninfected Group) to 86,478 genes. To normalize the data to avoid statistical bias, 24,097 genes from the mice with the lowest gene number were used as a baseline for normalization of all the sequences. OTU (97\% similarity) analysis was used to estimate richness, evenness and diversity of the bacterial communities. A total of 13,353 OTUs were obtained including 9,118 OTUs from mice infected with E. granulosus, and 8,423 OTUs from the uninfected mice (Table 2). No significant difference was evident between the two groups of mice in term of OTU numbers $(p>0.05)$. The Good's coverage was over $93.5 \%$ (93.5 97.8\%) for each sample, and over 98\% for the two groups, respectively (Table 2 ), meaning that the sequencing depth was sufficient to undertake microbiota analysis with two groups.

\section{Core Microbiome in the Gut of Mice}

Ribosomal Database Project analysis showed that $99.7 \%$ of the $16 \mathrm{~S}$ rRNA genes were aligned into nine phyla with the common bacteria Firmicutes, Bacteroidetes and Proteobacteria being dominant in both infected and uninfected groups. RDP analysis clustered $93.5 \%$ of the genes (OTUs) into 58 families and 13 families were identified as the major taxa and core microbiomes co-existing in the two groups. The genes in those families accounted for 91.61 and $94.27 \%$ of the microbiome community in the infected group and uninfected group, respectively (Table 3). Among the 13 families, Lachnospiraceae was mostly predominant in both groups, accounting for 41.42 and $43.92 \%$ of the total microbiome, respectively. Ruminococcaceae and

TABLE 3 | The major families of microbiota in E. granulosus infected mice and uninfected mice.

\begin{tabular}{lccc}
\hline Family & Uninfected (\%) & Infected (\%) & $\boldsymbol{p}$-value \\
\hline Lachnospiraceae & 41.42 & 43.92 & $p>0.05$ \\
Porphyromonadaceae & 14.21 & 15.75 & $p>0.05$ \\
Ruminococcaceae & 11.52 & 10.87 & $p>0.05$ \\
Rikenellaceae & 6.61 & 5.33 & $p>0.05$ \\
Bacteroidaceae & 5.80 & 5.93 & $p>0.05$ \\
Helicobacteraceae & 3.31 & 3.94 & $p>0.05$ \\
Prevotellaceae & 2.32 & 1.94 & $p>0.05$ \\
Desulfovibrionaceae & 1.94 & 2.30 & $p>0.05$ \\
Coriobacteriaceae & 0.14 & 0.20 & $p>0.05$ \\
Deferribacteraceae & 1.82 & 2.73 & $p>0.05$ \\
Lactobacillaceae & 1.80 & 0.90 & $p>0.05$ \\
Enterobacteriaceae & 0.39 & 0.17 & $p>0.05$ \\
Erysipelotrichaceae & 0.33 & 0.29 & $p>0.05$ \\
\hline Unifected & & & $p$
\end{tabular}

Uninfected, uninfected mice; Infected, mice infected with E. granulosus. 


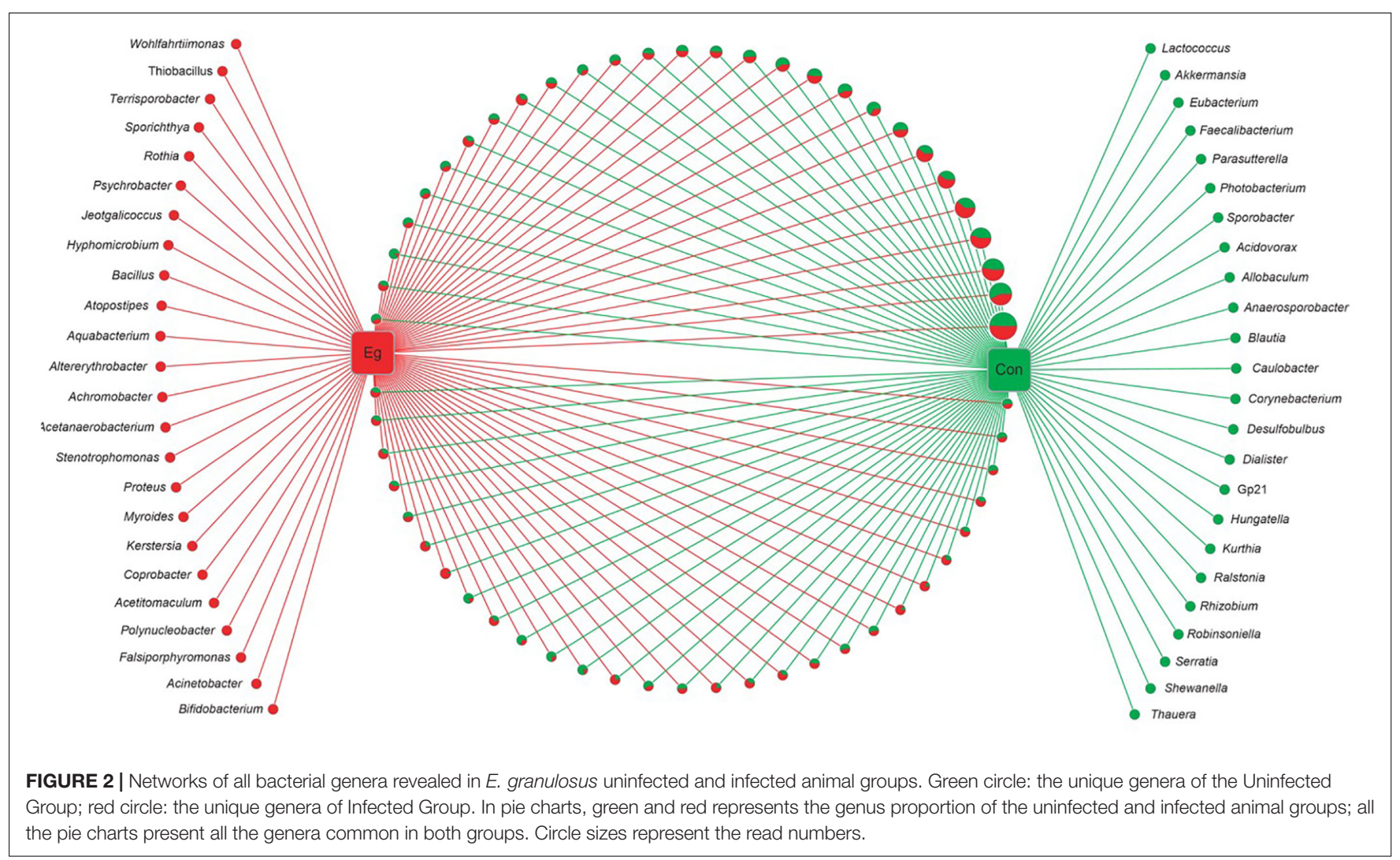

Porphyromonadaceae were also dominant ( $>10 \%$ of the entire microbiome in both groups).

Among the 58 families, $40.4 \%$ of genes (OUTs) have genus reference information and were aligned into 105 classified genera (Figure 2). There were 57 genera co-existing in both groups. However, there were 24 genera present in the Infection Group and another 24 genera in the Uninfected Group. The proportion of all the group unique genera was less than $0.01 \%$, and no significant differences were found between the two groups.

Among the 105 classified genera, 33 were core genera (with each genus comprising $>0.1 \%$ of total the microbiome), including Bacteroides, Odoribacter, Clostridium XlVa, Helicobacter, Alistipes, Barnesiella and Mucispirillum (Table 4). Among the predominant genera, there were 27 ubiquitous (core) genera which were consistently found in all samples and comprised more than $38 \%$ of the total microbiome.

At the OTU level, there were significant differences between the two groups; 101 OTUs were significantly increased and 49 OTU were significantly decreased in the infected mouse group $(p<0.05)$ (Table 5). Of note, most (59.6\%) OTUs were unclassified into genera as no classification information is available for these OTUs.

\section{Bacterial Composition in Different Groups}

LEfSe analysis showed the composition of the bacterial populations in the guts of the infected and uninfected mice was similar, whereas richness, evenness and diversity were only slightly changed (Table 2 and Figure 3). In contrast, AMOVA analysis showed significant difference between the two groups for the microbiota $\left(P_{\mathrm{AMOVA}}=0.029\right)$. Species richness (OTU, ACE and Chao index) was higher in the E. granulosus Infected Group, and the evenness (Shannoneven and Simpsoneven) was lower in this group compared with the uninfected mice. As richness and evenness combined, there was no significant difference in the diversity between the two groups $(p>0.5)$. Consequently, the results of these analyses indicated that E. granulosus infection did not alter much of the composition of the core bacteria present in the mouse gut significantly, although some rare bacteria in very low abundance were increased.

Among the 13 core families, LEfSe analysis showed no significant difference between the E. granulosus Infected Group and Uninfected Group in terms of microbiome. Among the major abundant genera, three showed significant differences between the groups (Table 4 , LDA $>2, p<0.05$ ). The infected mice significantly increases two genera, including Eisenbergiella (1.9 times) and Parabacteroides (17.5 times) compared with the uninfected mice $(p<0.05)$.

\section{Predicted Functional Potential Changes in the Microbiomes of the $E$. granulosus Infection and Uninfected Groups}

We used PICRUSt to predict and compare the microbial functional potential changes between the two groups. A total of 47 Kos were found to be significantly increased in the Infected 
TABLE 4 | The abundance of genera (>0.1\%) in the gut microbiomes of E. granulosus infected mice and uninfected mice determined by LEfSe analysis.

\begin{tabular}{|c|c|c|c|c|c|c|}
\hline Order & Family & Genus & Feature & Uninfected (\%) & Infected (\%) & $p$-value \\
\hline Coriobacteriales & Coriobacteriaceae & Enterorhabdus & Ubiquitous & 0.06 & 0.08 & \\
\hline Bacteroidales & Bacteroidaceae & Bacteroides & Ubiquitous & 5.80 & 5.93 & \\
\hline Bacteroidales & Porphyromonadaceae & Barnesiella & Ubiquitous & 2.06 & 2.99 & \\
\hline Bacteroidales & Porphyromonadaceae & Odoribacter & Ubiquitous & 3.86 & 4.33 & \\
\hline Bacteroidales & Porphyromonadaceae & Parabacteroides & Ubiquitous & $0.29^{*}$ & $0.56^{*}$ & 0.023976 \\
\hline Bacteroidales & Prevotellaceae & Alloprevotella & Ubiquitous & 1.55 & 1.13 & \\
\hline Bacteroidales & Prevotellaceae & Prevotella & Ubiquitous & 0.73 & 0.80 & \\
\hline Bacteroidales & Rikenellaceae & Rikenella & Ubiquitous & 1.81 & 1.67 & \\
\hline Deferribacterales & Deferribacteraceae & Mucispirillum & Ubiquitous & 1.81 & 2.72 & \\
\hline Lactobacillales & Lactobacillaceae & Lactobacillus & Ubiquitous & 1.80 & 0.90 & \\
\hline Clostridiales & Clostridiaceae 1 & Clostridium sensu stricto & & 0.12 & 0.17 & \\
\hline Clostridiales & Lachnospiraceae & Acetatifactor & Ubiquitous & 1.10 & 1.01 & \\
\hline Clostridiales & Lachnospiraceae & Clostridium XIVa & Ubiquitous & 2.70 & 4.19 & \\
\hline Clostridiales & Lachnospiraceae & Clostridium XIVb & Ubiquitous & 0.40 & 0.28 & \\
\hline Clostridiales & Lachnospiraceae & Dorea & Ubiquitous & 0.10 & 0.07 & \\
\hline Clostridiales & Lachnospiraceae & Eisenbergiella & & $0.02 *$ & $0.35^{*}$ & 0.001998 \\
\hline Clostridiales & Lachnospiraceae & Lachnospiraceae incertae_sedis & Ubiquitous & 0.56 & 0.61 & \\
\hline Clostridiales & Lachnospiraceae & Roseburia & Ubiquitous & 0.06 & 0.11 & \\
\hline Clostridiales & Ruminococcaceae & Anaerotruncus & Ubiquitous & 0.31 & 0.31 & \\
\hline Clostridiales & Ruminococcaceae & Butyricicoccus & Ubiquitous & 0.35 & 0.35 & \\
\hline Clostridiales & Ruminococcaceae & Clostridium IV & Ubiquitous & 0.04 & 0.05 & \\
\hline Clostridiales & Ruminococcaceae & Flavonifractor & Ubiquitous & 1.62 & 1.78 & \\
\hline Clostridiales & Ruminococcaceae & Intestinimonas & Ubiquitous & $0.15^{*}$ & $0.11^{*}$ & 0.035964 \\
\hline Clostridiales & Ruminococcaceae & Oscillibacter & Ubiquitous & 1.69 & 1.29 & \\
\hline Clostridiales & Ruminococcaceae & Pseudoflavonifractor & Ubiquitous & 0.68 & 0.73 & \\
\hline Clostridiales & Ruminococcaceae & Ruminococcus & & 0.13 & 0.02 & \\
\hline Erysipelotrichales & Erysipelotrichaceae & Erysipelotrichaceae incertae_sedis & Ubiquitous & 0.21 & 0.14 & \\
\hline Erysipelotrichales & Erysipelotrichaceae & Turicibacter & & 0.03 & 0.10 & \\
\hline Desulfovibrionales & Desulfovibrionaceae & Bilophila & & 0.03 & 0.03 & \\
\hline Desulfovibrionales & Desulfovibrionaceae & Desulfovibrio & Ubiquitous & 0.34 & 0.67 & \\
\hline Campylobacterales & Helicobacteraceae & Helicobacter & Ubiquitous & 3.31 & 3.94 & \\
\hline Enterobacteriales & Enterobacteriaceae & Escherichia/Shigella & Ubiquitous & 0.39 & 0.17 & \\
\hline Anaeroplasmatales & Anaeroplasmataceae & Anaeroplasma & & 0.16 & 0.10 & \\
\hline
\end{tabular}

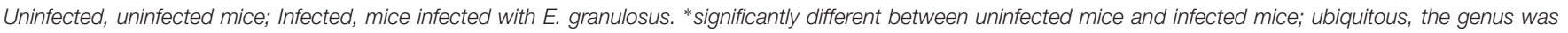
identified as present in all samples.

Group ( $p<0.05$ ) (Table 6). At KEGG level 3, 7 pathways were identified as being significant difference $(p<0.05)$ between the Infected Group and Uninfected Group, and the changed pathways belonged to the metabolism category, including "Biotin metabolism," "Biosynthesis and Biodegradation of secondary metabolites," "Ether lipid metabolism," "Steroid biosynthesis," "Aminobenzoate degradation," "Tryptophan metabolism" and "Limonene and pinene degradation."

\section{Correlations Between Bacterial Composition and Immunoglobulin Isotypes}

Spearman correlation analysis showed strong correlations of IgG, IgG1 and IgG2a with nine major genera (Table 7 and Figure 4). The numbers of Enterorhabdus, Barnesiella and Clostridium XlVa were positively correlated with IgG1, IgG2a and IgG2b levels, respectively. IgA was positively associated with increased numbers of genera Clostridium IV, Lachnospiraceae Incertae sedis and Mucispirillum. In addition, IgG, IgG1, IgG2b and IgG3 were associated with decreased numbers of genera Escherichia/Shigella, Ruminococcus, Ruminococcus/Intestinimonas and Ruminococcus, respectively (Table 7 ).

\section{DISCUSSION}

The ecological balance of the microbiota in the gut is crucial for maintaining healthy condition (Cani et al., 2008). Disruption of the balance of the gut microbiota is associated with a range of diseases, including colorectal cancer, autoimmune diseases, metabolic diseases, among others (Sokol et al., 2008; Jiang et al., 2015). In this study, we showed that E. granulosus infection increased two genera of gut microbiota: Eisenbergiella and genus 
TABLE 5 | The abundance of OTU $(p<0.01)$ in the infected mice and uninfected mice determined by LEfSe analysis.

\begin{tabular}{|c|c|c|c|c|}
\hline \#OTU & Taxonomy & Uninfected: mean rel.freq.(\%) & Infected: mean rel.freq.(\%) & $p$-values \\
\hline OTU00452 & Clostridiales;Lachnospiraceae;Clostridium XIVa & 0.0139587 & 0.04387 & 0.000351 \\
\hline OTU00048 & Desulfovibrionales;Desulfovibrionaceae;unclassified_Desulfovibrionaceae & 0.0067907 & 0.934319 & 0.000472 \\
\hline OTU00409 & Clostridiales;Lachnospiraceae;Acetatifactor & 0.0033954 & 0.040906 & 0.001051 \\
\hline OTU00832 & Desulfovibrionales;Desulfovibrionaceae;unclassified_Desulfovibrionaceae & 0 & 0.013043 & 0.001199 \\
\hline OTU01146 & Clostridiales;Lachnospiraceae;unclassified_Lachnospiraceae & 0.0075453 & 0.000296 & 0.001367 \\
\hline OTU01119 & Clostridiales;Lachnospiraceae;unclassified_Lachnospiraceae & 0.0003773 & 0.005336 & 0.001834 \\
\hline OTU00050 & Bacteroidales;Porphyromonadaceae;Parabacteroides & 0.1011065 & 0.450856 & 0.002202 \\
\hline OTU00186 & Clostridia;Clostridiales;unclassified_Clostridiales & 0.1844817 & 0.046538 & 0.00282 \\
\hline OTU00122 & Clostridiales;Lachnospiraceae;Eisenbergiella & 0.0211267 & 0.335252 & 0.002849 \\
\hline OTU00410 & Clostridiales;Ruminococcaceae;Oscillibacter & 0.0045272 & 0.034681 & 0.003348 \\
\hline OTU00058 & Campylobacterales;Helicobacteraceae;Helicobacter & 0.0580985 & 0.596103 & 0.003788 \\
\hline OTU00720 & Clostridia;Clostridiales;unclassified_Clostridiales & 0.0015091 & 0.01245 & 0.00456 \\
\hline OTU01984 & Desulfovibrionales;Desulfovibrionaceae;unclassified_Desulfovibrionaceae & 0 & 0.002668 & 0.006591 \\
\hline OTU01307 & Clostridiales;Lachnospiraceae;unclassified_Lachnospiraceae & 0.0003773 & 0.004743 & 0.007635 \\
\hline OTU01051 & Bacteria;Firmicutes;unclassified_Firmicutes & 0.0003773 & 0.007411 & 0.007741 \\
\hline OTU02591 & Desulfovibrionales;Desulfovibrionaceae;unclassified_Desulfovibrionaceae & 0 & 0.001779 & 0.008089 \\
\hline OTU01588 & Bacteroidales;Porphyromonadaceae;Odoribacter & 0.0033954 & 0.000296 & 0.008438 \\
\hline OTU00715 & Desulfovibrionales;Desulfovibrionaceae;unclassified_Desulfovibrionaceae & 0.0120724 & 0.003261 & 0.008709 \\
\hline OTU00460 & Clostridiales;Lachnospiraceae;unclassified_Lachnospiraceae & 0.0049044 & 0.049206 & 0.009747 \\
\hline
\end{tabular}

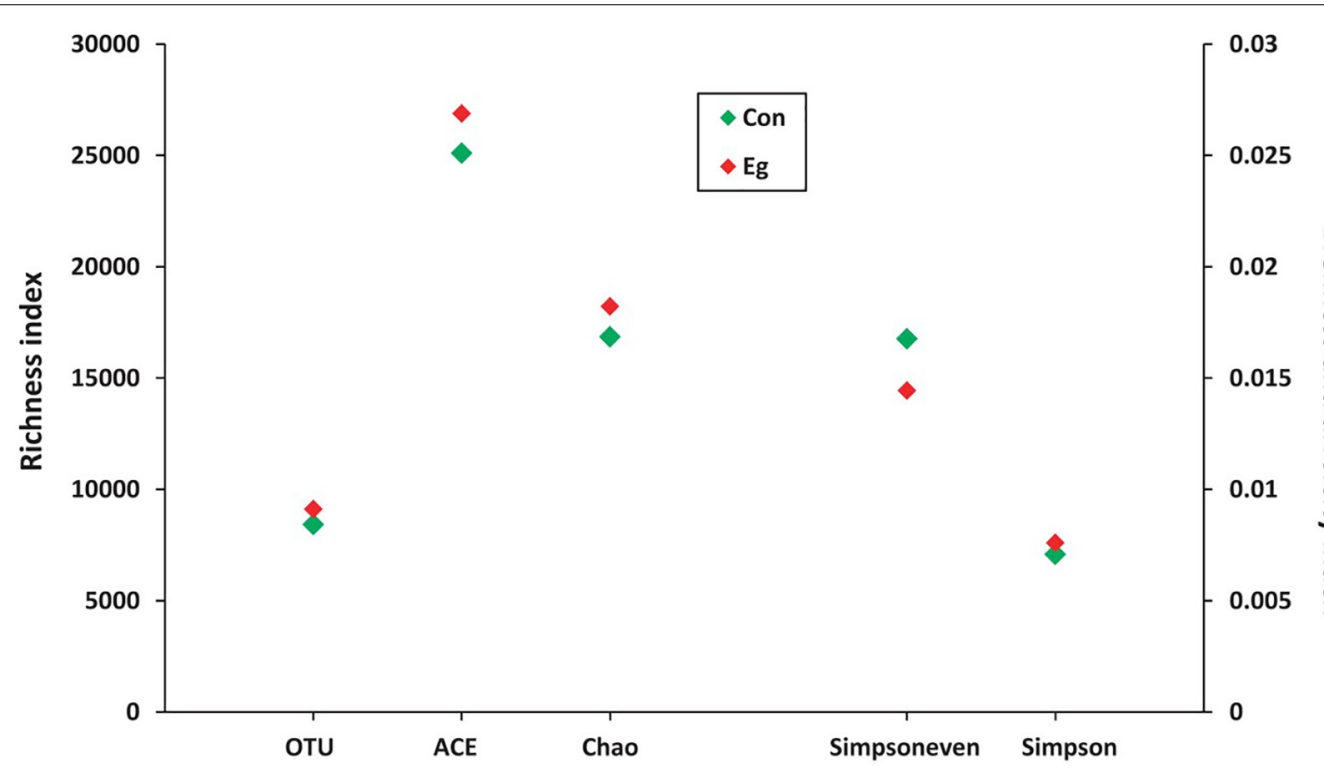

FIGURE 3 | The microbial diversity variability in mice infected (Infected Group) or uninfected (Uninfected Group) with E. granulosus. LEfSe analysis showed the composition of the bacterial populations in the guts of the infected and uninfected mice was similar, whereas richness, evenness and diversity were no changed.

Parabacteroides, with most genera remaining unchange. The two genera are in the family Lachnospiraceae. Their increase may impact on human health (Plieskatt et al., 2013), and may be associated with diabetes in mice (Kameyama and Itoh, 2014).

At the OTU level, there were 150 OTUs significantly changed in the infected mice. However, among the OTUs, only 49 OTUs have taxonomic information at genus level with 101 without predicted taxonomic classification information, which limited our further analysis (Table 5). Additionally, the LEfSe analysis also showed genera Eisenbergiella and Parabacteroides increased in the infected mice, suggesting that these two genera of bacteria might be biomarkers for E. granulosus infection. In our study, the genus Eisenbergiella in the family Lachnospiraceae was upregulated significantly in the infected mice, however, there is very limited biological function information on this genus. Combined with antibody analysis in this study, this genus of bacteria may be associated with a Th2 response.

Another increased genus in Infected Group is Parabacteroides whereas species belonging to the genus Parabacteroides are saccharolytic (Rajilic-Stojanovic and de Vos, 2014), being 
TABLE 6 | Functional predictions using the PICRUSt base on 16S rRNA gene copy numbers.

\begin{tabular}{|c|c|c|c|c|}
\hline KO accession & Annotation & Infected & Uninfected & $p$-value \\
\hline K00655 & 1-acyl-sn-glycerol-3-phosphate acyltransferase [EC:2.3.1.51] & 17,607 & 15,826 & 0.01887 \\
\hline K02312 & 2,3-dihydroxybenzoate-AMP ligase [EC:2.7.7.58] & 4,214 & 3,074 & $2.43 E-02$ \\
\hline K01826 & 5-carboxymethyl-2-hydroxymuconate isomerase [EC:5.3.3.10] & 2 & 0 & 0.015531 \\
\hline K01905 & Acetyl-CoA synthetase (ADP-forming) [EC:6.2.1.13] & 4,216 & 3,063 & 0.02436 \\
\hline K12554 & Alanine adding enzyme [EC:2.3.2.-] & 1 & 0 & 0.020538 \\
\hline K05341 & Amylosucrase [EC:2.4.1.4] & 4,223 & 3,068 & 0.023681 \\
\hline K03325 & Arsenite transporter, ACR3 family & 5,022 & 3,773 & 1.16E-02 \\
\hline K01338 & ATP-dependent Lon protease [EC:3.4.21.53] & 15,636 & 13,406 & 0.023016 \\
\hline K01305 & Beta-aspartyl-dipeptidase (metallo-type) [EC:3.4.19.-] & 4,245 & 3,082 & 0.023369 \\
\hline K01012 & Biotin synthetase [EC:2.8.1.6] & 15,209 & 12,941 & 0.013677 \\
\hline K03519 & Carbon-monoxide dehydrogenase medium subunit [EC:1.2.99.2] & 4,219 & 3,067 & 0.024608 \\
\hline K01647 & Citrate synthase [EC:2.3.3.1] & 14,507 & 12,412 & 0.023345 \\
\hline K01739 & Cystathionine gamma-synthase [EC:2.5.1.48] & 4,250 & 3,105 & 0.023501 \\
\hline K00901 & Diacylglycerol kinase [EC:2.7.1.107] & 5,333 & 4,032 & 0.012241 \\
\hline K11754 & Dihydrofolate synthase/folylpolyglutamate synthase [EC:6.3.2.12 6.3.2.17] & 26,899 & 24,234 & 0.011904 \\
\hline K07458 & DNA mismatch endonuclease, patch repair protein [EC: 3.1.-.-] & 4,883 & 3,692 & 0.01095 \\
\hline K06212 & Formate transporter & 4,268 & 3,116 & 0.021367 \\
\hline K13892 & Glutathione transport system ATP-binding protein & 4,226 & 3,072 & 0.024579 \\
\hline K04653 & Hydrogenase expression/formation protein HypC & 7,620 & 6,473 & 0.017468 \\
\hline K04654 & Hydrogenase expression/formation protein HypD & 7,624 & 6,475 & 0.016744 \\
\hline K04655 & Hydrogenase expression/formation protein HypE & 7,696 & 6,567 & 0.019062 \\
\hline K04656 & Hydrogenase maturation protein HypF & 7,630 & 6,479 & 0.016758 \\
\hline K04652 & Hydrogenase nickel incorporation protein HypB & 7,888 & 6,740 & 0.021211 \\
\hline K09384 & Hypothetical protein & 4,225 & 3,076 & $2.37 \mathrm{E}-02$ \\
\hline K09703 & Hypothetical protein & 4,217 & 3,064 & 0.024347 \\
\hline K07301 & Inner membrane protein & 8,454 & 7,324 & 0.000592 \\
\hline K03779 & $\mathrm{L}(+)$-tartrate dehydratase alpha subunit [EC:4.2.1.32] & 4,264 & 3,106 & 0.024966 \\
\hline K00879 & L-fuculokinase [EC:2.7.1.51] & 4,246 & 3,099 & 0.023788 \\
\hline K08369 & MFS transporter, putative metabolite:H+ symporter & 4,280 & 3,112 & 0.021188 \\
\hline K02018 & Molybdate transport system permease protein & 10,849 & 8,353 & $2.09 \mathrm{E}-02$ \\
\hline K03637 & Molybdenum cofactor biosynthesis protein C & 5,564 & 4,311 & 0.024072 \\
\hline K07474 & Phage terminase small subunit & 4,318 & 3,190 & 0.022446 \\
\hline K02759 & PTS system, cellobiose-specific IIA component [EC:2.7.1.69] & 4,336 & 3,207 & 0.022898 \\
\hline K02760 & PTS system, cellobiose-specific IIB component [EC:2.7.1.69] & 4,448 & 3,372 & 0.019282 \\
\hline K02777 & PTS system, glucose-specific IIA component [EC:2.7.1.69] & 4,677 & 3,516 & 0.016117 \\
\hline K10026 & Queuosine biosynthesis protein QueE & 4,760 & 3,668 & $2.47 \mathrm{E}-02$ \\
\hline K12996 & Rhamnosyltransferase [EC:2.4.1.-] & 4,219 & 3,080 & 0.024267 \\
\hline K03086 & RNA polymerase primary sigma factor & 17,918 & 16,008 & $3.41 E-03$ \\
\hline K05297 & Rubredoxin-NAD+ reductase [EC:1.18.1.1] & 4,352 & 3,216 & 0.020307 \\
\hline K03438 & S-adenosyl-methyltransferase [EC:2.1.1.-]; 16S rRNA (cytosine1402-N4)-methyltransferase [EC:2.1.1.199] & 16,924 & 15,121 & 0.014162 \\
\hline K07313 & Serine/threonine protein phosphatase 1 [EC:3.1.3.16] & 5,067 & 3,888 & 0.010963 \\
\hline K02945 & Small subunit ribosomal protein S1 & 16,311 & 14,363 & $1.68 \mathrm{E}-03$ \\
\hline K05814 & sn-glycerol 3-phosphate transport system permease protein & 4,248 & 3,118 & 0.023484 \\
\hline K11928 & Sodium/proline symporter & 4,419 & 3,257 & 0.022096 \\
\hline K01695 & Tryptophan synthase alpha chain [EC:4.2.1.20] & 9,531 & 8,500 & 0.021878 \\
\hline K07665 & Two-component system, OmpR family, copper resistance phosphate regulon response regulator CusR & 7,719 & 6,661 & 0.012516 \\
\hline K04784 & Yersiniabactin non-ribosomal peptide synthetase & 4,212 & 3,062 & 0.024426 \\
\hline Average & & 2,156 & 2,116 & \\
\hline
\end{tabular}

The numbers of Infected and Uninfected were the normalization of copy numbers. Uninfected, uninfected mice; Infected, mice infected with E. granulosus.

producers of short chain fatty acids (SCFAs) including acetate, propionate and butyrate as bacterial fermentation products (Cummings et al., 1987; Correa-Oliveira et al., 2016; Lloyd-Price et al., 2016). SCFAs act as links between the microbiota and the host immune system (Correa-Oliveira et al., 2016). The liver is the major systemic organ for SCFA metabolism and consumption 
TABLE 7 | Genera of bacteria correlating highly with serum antibody isotypes in E. granulosus infected mice and uninfected mice.

\begin{tabular}{|c|c|c|c|c|c|c|c|}
\hline Genus & Uninfected (\%) & Infected (\%) & Factor & Uninfected & Infected & Spearman Coef & $p$-value \\
\hline Barnesiella & 2.06 & 2.99 & $\lg G 2 a$ & 0.2758 & 0.8682 & 0.9 & 0.037386 \\
\hline Ruminococcus & 0.13 & 0.02 & $\lg G 1$ & 0.2801 & 3.4034 & -0.9 & 0.037386 \\
\hline Ruminococcus & 0.13 & 0.02 & $\lg G 2 b$ & 0.2434 & 0.8148 & -0.9 & 0.037386 \\
\hline Ruminococcus & 0.13 & 0.02 & $\operatorname{lgG} 3$ & 0.2329 & 1.0469 & -1 & 0 \\
\hline Ruminococcus & 0.13 & 0.02 & $\operatorname{lgM}$ & 0.3879 & 2.7058 & -0.9 & 0.037386 \\
\hline Clostridium IV & 0.04 & 0.05 & $\lg A$ & 0.1563 & 0.3758 & 0.9747 & 0.004818 \\
\hline Enterorhabdus & 0.06 & 0.08 & $\lg \mathrm{G} 1$ & 0.2801 & 3.4034 & 0.9 & 0.037386 \\
\hline Intestinimonas & 0.15 & 0.11 & $\lg G 2 b$ & 0.2434 & 0.8148 & -0.9 & 0.037386 \\
\hline Escherichia/Shigella & 0.39 & 0.17 & $\lg G$ & 0.7014 & 3.9271 & -1 & 0 \\
\hline Lachnospiracea Incertaesedis & 0.56 & 0.61 & $\lg A$ & 0.1564 & 0.3758 & 0.9747 & 0.004818 \\
\hline Mucispirillum & 1.81 & 2.72 & $\lg \mathrm{A}$ & 0.1564 & 0.3758 & 0.9747 & 0.004818 \\
\hline Clostridium XIVa & 2.70 & 4.19 & $\lg G 2 b$ & 0.2434 & 0.8148 & 0.9 & 0.037386 \\
\hline
\end{tabular}

Uninfected, uninfected mice; Infected, mice infected with E. granulosus.

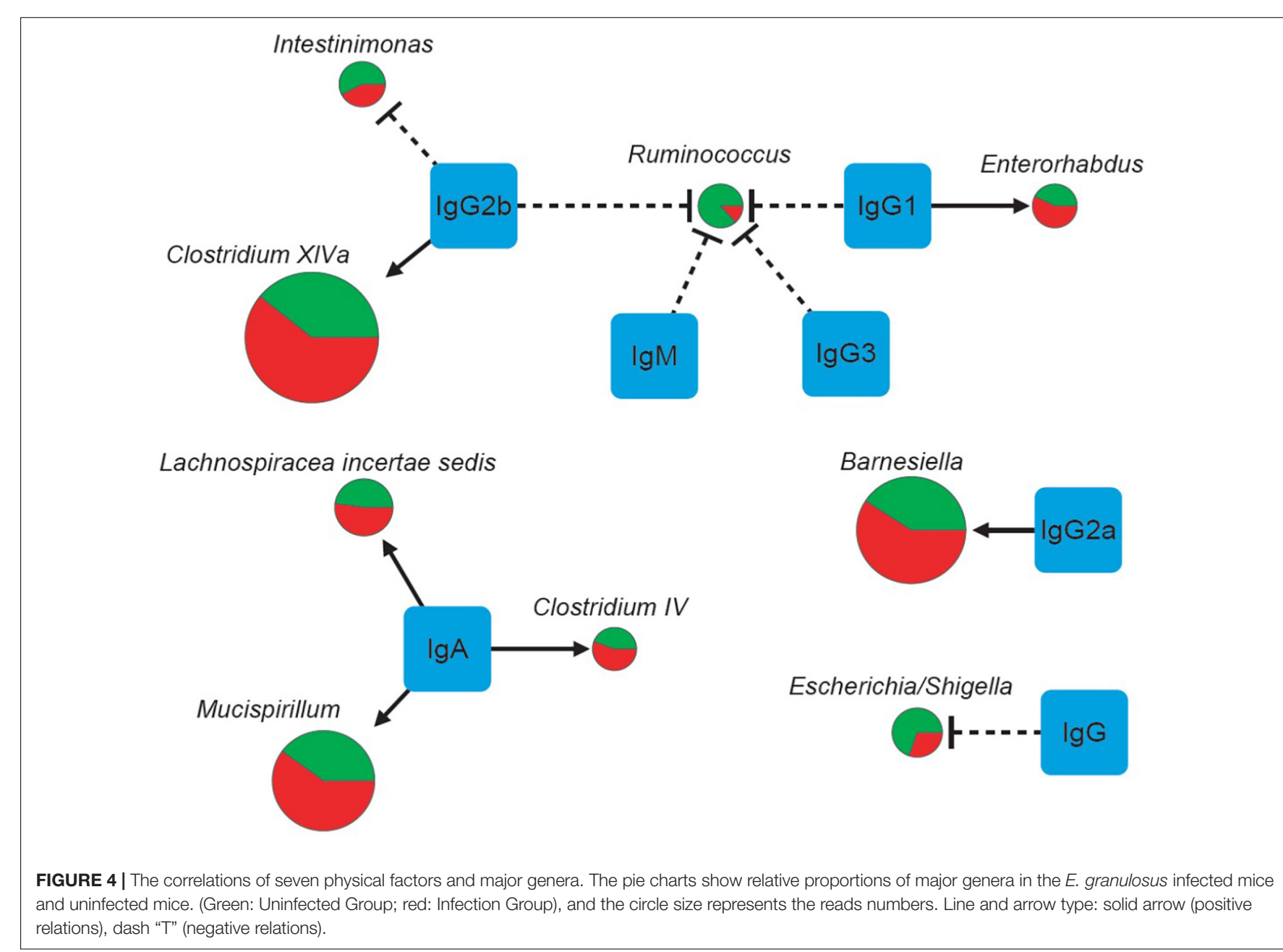

(Kim et al., 2014), SCFAs released by the gut and equaled by hepatic uptake (Bloemen et al., 2009). Parabacteroides has evolved to contain a gene encoding a major capsid protein (Rosenwald et al., 2014) one of the phage orthologous groups (Kristensen et al., 2013). One report demonstrated that Parabacteroides was prevalent in diabetic (Wu et al., 2010). The increasing of genus
Parabacteroides in hydatid infection may be associated with hepatic alteration.

Functional predictions showed seven pathways of the gut microbiota in the E. granulosus infection group were altered compared with the uninfected group. These pathways included biotin, lipid metabolism, and tryptophan metabolism. 
The synergistic effect of bacteria leads to the difference of gut flora metabolic pathways due to some or all intestinal bacteria involving in metabolism. Biotin metabolism in the intestine is regulated through transcriptional and posttranscriptional mechanisms. Its balance plays a key role in regulating the absorption and the function of biotin in tissues (Zoetendal et al., 2012). Based on pathway impact analysis, we found that tryptophan metabolism was decreased in the E. granulosus infection group. In mice infected with schistosomes, tryptophan or compounds from tryptophan metabolism were up-regulated and increased in urine which indicate possible problems in tryptophan metabolism in these infected animals (Njagi et al., 1992; Wang et al., 2004).

We showed that the bacterial composition of nine major genera had strong correlations with the levels of $\operatorname{IgG}$, IgG1 and IgG2a antibodies against HCF antigens (Figure 4). The numbers of genera Enterorhabdus, and Clostridium XlVa were positively correlated with IgG1 and IgG2b levels, indicating that these bacteria can be tolerated with those Th2 associated antibodies or Th2 responses may benefit those genera of bacteria. Meanwhile, IgG2a, a Th1 associated antibody, was associated with increased number of genus Barnesiella, indicating Th1 has a role for increasing genus Barnesiella. Our data also showed that Th2 associated antibodies IgG1 and IgG2b and IgG3 decreased numbers of genera Escherichia/Shigella, and Ruminococcus, Ruminococcus/Intestinimonas (Table 7 and Figure 4). Interestingly, Intestinimonas decreased significantly in E. granulosus infection group as a differential genus by LEfSe analysis, perhaps it is associated with some kinds of change, then we concluded that it is highly related to IgG2b by Spearman coefficient correlation analysis and is consistent with the immune background of E. granulosus infection. So IgG2b may play impartment role in inhibition of Intestinimonas. Clostridium has been found to be associated with a number of diseases. It showed that Clostridium may participate in antibiotic-associated diarrhea (Buffie et al., 2015) and damages the human intestine in vitro (Fernandez Miyakawa et al., 2005). Barnesiella is present in the healthy intestinal tract and is influenced by antibiotics, and intestinal colonization with Barnesiella confers resistance to intestinal domination and bloodstream infection with vancomycin-resistant Enterococcus (Ubeda et al., 2013).

\section{REFERENCES}

Bloemen, J. G., Venema, K., Van De Poll, M. C., Olde Damink, S. W., Buurman, W. A., and Dejong, C. H. (2009). Short chain fatty acids exchange across the gut and liver in humans measured at surgery. Clin. Nutr. 28, 657-661. doi: 10.1016/j.clnu.2009.05.011

Buffie, C. G., Bucci, V., Stein, R. R., Mckenney, P. T., Ling, L., Gobourne, A., et al. (2015). Precision microbiome reconstitution restores bile acid mediated resistance to Clostridium difficile. Nature 517, 205-208. doi: 10.1038/ nature 13828

Cani, P. D., Bibiloni, R., Knauf, C., Waget, A., Neyrinck, A. M., Delzenne, N. M., et al. (2008). Changes in gut microbiota control metabolic endotoxemiainduced inflammation in high-fat diet-induced obesity and diabetes in mice. Diabetes Metab. Res. Rev. 57, 1470-1481. doi: 10.2337/db07-1403
In summary, we explored gut microbiota in mice infected with E. granulosus, and found that chronic E. granulosus infection increased 101 OTUs including two genera of gut microbiota in mice. Functional prediction showed seven pathways of gut microbiota were altered, and bacterial composition of major genera had positive correlations with IgG1 and IgG2b in E. granulosus infected mice.

Whereas more than $85 \%$ of the genomic sequences between mouse and Homo are conserved, overall gene expression and its regulation are considerably different between the two species (Hugenholtz and de Vos, 2018). Human and mouse seems to be similar at phylum level, Bacteroidetes and Firmicutes are the two major bacterial phyla of the intestinal tract (Rawls et al., 2006). However, we do not know whether E. granulosus infection will affect human intestinal flora in the same way as the mouse and further studies are now required to understand the further possible mechanisms associated with altered colonization resistance after helminth infection and to determine changes in the gut microbiota of patients with CE.

\section{ACCESSION NUMBERS}

The sequence data have been submitted to the GeneBank Sequence Read Archive (Accession Number PRJNA396089).

\section{AUTHOR CONTRIBUTIONS}

WZ, JL, and DM contributed to conception and design of the study. JB organized the database. LH, WQ, and TW finished the animal experiments. HZ, ZZ, and YW performed the statistical analysis. JB and YW wrote the first draft of the manuscript. GG, XZ, and BG wrote sections of the manuscript. All authors contributed to manuscript revision, read and approved the submitted version.

\section{FUNDING}

The study was financially supported by The National Natural Science Foundation of China (NSFC 81460308 and NSFC U1303203).

Casaravilla, C., Pittini, A., Ruckerl, D., Seoane, P. I., Jenkins, S. J., Macdonald, A. S., et al. (2014). Unconventional maturation of dendritic cells induced by particles from the laminated layer of larval Echinococcus granulosus. Infect. Immun. 82, 3164-3176. doi: 10.1128/IAI.01959-14

Chen, Y. C., Greenbaum, J., Shen, H., and Deng, H. W. (2017). Association between gut microbiota and bone health: potential mechanisms and prospective. J. Clin. Endocrinol. Metab. 102, 3635-3646. doi: 10.1210/jc.2017-00513

Cole, J. R., Wang, Q., Cardenas, E., Fish, J., Chai, B., Farris, R. J., et al. (2009). The Ribosomal Database Project: improved alignments and new tools for rRNA analysis. Nucleic Acids Res. 37, D141-D145. doi: 10.1093/nar/ gkn879

Correa-Oliveira, R., Fachi, J. L., Vieira, A., Sato, F. T., and Vinolo, M. A. (2016). Regulation of immune cell function by short-chain fatty acids. Clin. Transl. Immunol. 5:e73. doi: 10.1038/cti.2016.17 
Cummings, J. H., Pomare, E. W., Branch, W. J., Naylor, C. P., and Macfarlane, G. T. (1987). Short chain fatty acids in human large intestine, portal, hepatic and venous blood. Gut 28, 1221-1227. doi: 10.1136/gut.28.10.1221

Elissondo, M., Ceballos, L., Dopchiz, M., Andresiuk, V., Alvarez, L., Bruni, S. S., et al. (2007). In vitro and in vivo effects of flubendazole on Echinococcus granulosus metacestodes. Parasitol. Res. 100, 1003-1009. doi: 10.1007/s00436006-0381-y

Fernandez Miyakawa, M. E., Pistone Creydt, V., Uzal, F. A., Mcclane, B. A., and Ibarra, C. (2005). Clostridium perfringens enterotoxin damages the human intestine in vitro. Infect. Immun. 73, 8407-8410. doi: 10.1128/IAI.73.12.84078410.2005

Gavidia, C. M., Gonzalez, A. E., Zhang, W., Mcmanus, D. P., Lopera, L., Ninaquispe, B., et al. (2008). Diagnosis of cystic echinococcosis, central Peruvian Highlands. Emerg. Infect. Dis. 14, 260-266. doi: 10.3201/eid1402. 061101

Gkouskou, K. K., Deligianni, C., Tsatsanis, C., and Eliopoulos, A. G. (2014). The gut microbiota in mouse models of inflammatory bowel disease. Front. Cell. Infect. Microbiol. 4:28. doi: 10.3389/fcimb.2014.00028

Gottstein, B. (2001). Animal models in parasitology. Contrib. Microbiol. 9, 31-44. doi: $10.1159 / 000060387$

Guernier, V., Brennan, B., Yakob, L., Milinovich, G., Clements, A. C., and Soares Magalhaes, R. J. (2017). Gut microbiota disturbance during helminth infection: can it affect cognition and behaviour of children? BMC Infect. Dis. 17:58. doi: 10.1186/s12879-016-2146-2

Hugenholtz, F., and de Vos, W. M. (2018). Mouse models for human intestinal microbiota research: a critical evaluation. Cell. Mol. Life Sci. 75, 149-160. doi: 10.1007/s00018-017-2693-8

Jensen, A. B., Ajslev, T. A., Brunak, S., and Sorensen, T. I. (2015). Long-term risk of cardiovascular and cerebrovascular disease after removal of the colonic microbiota by colectomy: a cohort study based on the Danish National Patient Register from 1996 to 2014. BMJ Open 5:e008702. doi: 10.1136/bmjopen-2015008702

Jiang, H., Ling, Z., Zhang, Y., Mao, H., Ma, Z., Yin, Y., et al. (2015). Altered fecal microbiota composition in patients with major depressive disorder. Brain Behav. Immun. 48, 186-194. doi: 10.1016/j.bbi.2015.03.016

Kameyama, K., and Itoh, K. (2014). Intestinal colonization by a Lachnospiraceae bacterium contributes to the development of diabetes in obese mice. Microbes Environ. 29, 427-430. doi: 10.1264/jsme2.ME14054

Kim, C. H., Park, J., and Kim, M. (2014). Gut microbiota-derived short-chain fatty acids, T cells, and inflammation. Immune Netw. 14, 277-288. doi: 10.4110/in. 2014.14.6.277

Kittelberger, R., Reichel, M. P., Jenner, J., Heath, D. D., Lightowlers, M. W., Moro, P., et al. (2002). Evaluation of three enzyme-linked immunosorbent assays (ELISAs) for the detection of serum antibodies in sheep infected with Echinococcus granulosus. Vet. Parasitol. 110, 57-76. doi: 10.1016/S03044017(02)00308-4

Kristensen, D. M., Waller, A. S., Yamada, T., Bork, P., Mushegian, A. R., and Koonin, E. V. (2013). Orthologous gene clusters and taxon signature genes for viruses of prokaryotes. J. Bacteriol. 195, 941-950. doi: 10.1128/JB.01801-12

Langille, M. G., Zaneveld, J., Caporaso, J. G., Mcdonald, D., Knights, D., Reyes, J. A., et al. (2013). Predictive functional profiling of microbial communities using 16S rRNA marker gene sequences. Nat. Biotechnol. 31, 814-821. doi: $10.1038 /$ nbt. 2676

Lloyd-Price, J., Abu-Ali, G., and Huttenhower, C. (2016). The healthy human microbiome. Genome Med. 8:51. doi: 10.1186/s13073-016-0307-y

McManus, D. P., Gray, D. J., Zhang, W., and Yang, Y. (2012). Diagnosis, treatment, and management of echinococcosis. BMJ 344:e3866. doi: 10.1136/bmj.e3866

Mourglia-Ettlin, G., Cucher, M., Arbildi, P., Rosenzvit, M., and Dematteis, S. (2016). Natural and induced antibodies contribute to differential susceptibility to secondary cystic echinococcosis of Balb/c and C57Bl/6 mice. Immunobiology 221, 103-115. doi: 10.1016/j.imbio.2015.07.016

Nakaya, K., Mamuti, W., Xiao, N., Sato, M. O., Wandra, T., Nakao, M., et al. (2006). Usefulness of severe combined immunodeficiency (scid) and inbred mice for studies of cysticercosis and echinococcosis. Parasitol. Int. 55(Suppl.), S91-S97. doi: 10.1016/j.parint.2005.11.014

Njagi, E. N., Bender, D. A., and Okelo, G. B. (1992). Tryptophan metabolism and vitamin B6 nutritional status in patients with schistosomiasis mansoni and in infected mice. Parasitology 104(Pt 3), 433-441. doi: 10.1017/ S0031182000063691

O'Keefe, S. J. (2016). Diet, microorganisms and their metabolites, and colon cancer. Nat. Rev. Gastroenterol. Hepatol. 13, 691-706. doi: 10.1038/nrgastro.2016.165

Parks, D. H., Tyson, G. W., Hugenholtz, P., and Beiko, R. G. (2014). STAMP: statistical analysis of taxonomic and functional profiles. Bioinformatics 30, 3123-3124. doi: 10.1093/bioinformatics/btu494

Plieskatt, J. L., Deenonpoe, R., Mulvenna, J. P., Krause, L., Sripa, B., Bethony, J. M., et al. (2013). Infection with the carcinogenic liver fluke Opisthorchis viverrini modifies intestinal and biliary microbiome. FASEB J. 27, 4572-4584. doi: 10.1096/fj.13-232751

Quast, C., Pruesse, E., Yilmaz, P., Gerken, J., Schweer, T., Yarza, P., et al. (2013). The SILVA ribosomal RNA gene database project: improved data processing and web-based tools. Nucleic Acids Res. 41, D590-D596. doi: 10.1093/nar/gks1219

Rajilic-Stojanovic, M., and de Vos, W. M. (2014). The first 1000 cultured species of the human gastrointestinal microbiota. FEMS Microbiol. Rev. 38, 996-1047. doi: 10.1111/1574-6976.12075

Ramanan, D., Bowcutt, R., Lee, S. C., Tang, M. S., Kurtz, Z. D., Ding, Y., et al. (2016). Helminth infection promotes colonization resistance via type 2 immunity. Science 352, 608-612. doi: 10.1126/science.aaf3229

Rawls, J. F., Mahowald, M. A., Ley, R. E., and Gordon, J. I. (2006). Reciprocal gut microbiota transplants from zebrafish and mice to germ-free recipients reveal host habitat selection. Cell 127, 423-433. doi: 10.1016/j.cell.2006.08.043

Rigano, R., Profumo, E., and Siracusano, A. (1997). New perspectives in the immunology of Echinococcus granulosus infection. Parassitologia 39, 275-277.

Rosenwald, A. G., Murray, B., Toth, T., Madupu, R., Kyrillos, A., and Arora, G. (2014). Evidence for horizontal gene transfer between Chlamydophila pneumoniae and Chlamydia phage. Bacteriophage 4:e965076. doi: 10.4161/ 21597073.2014.965076

Schloss, P. D., Gevers, D., and Westcott, S. L. (2011). Reducing the effects of PCR amplification and sequencing artifacts on $16 \mathrm{~S}$ rRNA-based studies. PLoS One 6:e27310. doi: 10.1371/journal.pone.0027310

Segata, N., Izard, J., Waldron, L., Gevers, D., Miropolsky, L., Garrett, W. S., et al. (2011). Metagenomic biomarker discovery and explanation. Genome Biol. 12:R60. doi: 10.1186/gb-2011-12-6-r60

Sokol, H., Pigneur, B., Watterlot, L., Lakhdari, O., Bermudez-Humaran, L. G., Gratadoux, J. J., et al. (2008). Faecalibacterium prausnitzii is an antiinflammatory commensal bacterium identified by gut microbiota analysis of Crohn disease patients. Proc. Natl. Acad. Sci. U.S.A. 105, 16731-16736. doi: 10.1073/pnas.0804812105

Sonnenburg, J. L., and Backhed, F. (2016). Diet-microbiota interactions as moderators of human metabolism. Nature 535, 56-64. doi: 10.1038/ nature 18846

Taylor, R. (1990). Interpretation of the correlation coefficient: a basic review. J. Diagn. Med. Sonogr. 6, 35-39. doi: 10.1177/875647939000600106

Thaiss, C. A., Zmora, N., Levy, M., and Elinav, E. (2016). The microbiome and innate immunity. Nature 535, 65-74. doi: 10.1038/nature 18847

Ubeda, C., Bucci, V., Caballero, S., Djukovic, A., Toussaint, N. C., Equinda, M., et al. (2013). Intestinal microbiota containing Barnesiella species cures vancomycin-resistant Enterococcus faecium colonization. Infect. Immun. 81, 965-973. doi: 10.1128/IAI.01197-12

Wang, H., Li, J., Guo, B., Zhao, L., Zhang, Z., Mcmanus, D. P., et al. (2016). In vitro culture of Echinococcus multilocularis producing protoscoleces and mouse infection with the cultured vesicles. Parasit. Vectors 9:411. doi: 10.1186/ s13071-016-1687-y

Wang, H., Li, J., Pu, H., Hasan, B., Ma, J., Jones, M. K., et al. (2014). Echinococcus granulosus infection reduces airway inflammation of mice likely through enhancing IL-10 and down-regulation of IL-5 and IL-17A. Parasit. Vectors 7:522. doi: 10.1186/s13071-014-0522-6

Wang, Q., Garrity, G. M., Tiedje, J. M., and Cole, J. R. (2007). Naive Bayesian classifier for rapid assignment of rRNA sequences into the new bacterial taxonomy. Appl. Environ. Microbiol. 73, 5261-5267. doi: 10.1128/AEM.000 62-07

Wang, W., Li, J., Yao, J., Wang, T., Li, S., Zheng, X., et al. (2017). In vitro and in vivo efficacies of novel carbazole aminoalcohols in the treatment of cystic echinococcosis. J. Antimicrob. Chemother. 72, 3122-3130. doi: 10.1093/jac/ $\mathrm{dkx} 250$ 
Wang, Y., Holmes, E., Nicholson, J. K., Cloarec, O., Chollet, J., Tanner, M., et al. (2004). Metabonomic investigations in mice infected with Schistosoma mansoni: an approach for biomarker identification. Proc. Natl. Acad. Sci. U.S.A. 101, 12676-12681. doi: 10.1073/pnas.040487 8101

Wegener Parfrey, L., Jirku, M., Sima, R., Jalovecka, M., Sak, B., Grigore, K., et al. (2017). A benign helminth alters the host immune system and the gut microbiota in a rat model system. PLoS One 12:e182205. doi: 10.1371/journal. pone.0182205

Wu, X., Ma, C., Han, L., Nawaz, M., Gao, F., Zhang, X., et al. (2010). Molecular characterisation of the faecal microbiota in patients with type II diabetes. Curr. Microbiol. 61, 69-78. doi: 10.1007/s00284-010-9582-9

Yu, L. C., Wei, S. C., and Ni, Y. H. (2018). Impact of microbiota in colorectal carcinogenesis: lessons from experimental models. Intest. Res. 16, 346-357. doi: 10.5217/ir.2018.16.3.346

Zeng, M. Y., Inohara, N., and Nunez, G. (2017). Mechanisms of inflammationdriven bacterial dysbiosis in the gut. Mucosal Immunol. 10, 18-26. doi: 10.1038/ mi.2016.75

Zhang, W., Li, J., and Mcmanus, D. P. (2003). Concepts in immunology and diagnosis of hydatid disease. Clin. Microbiol. Rev. 16, 18-36. doi: 10.1128/CMR. 16.1.18-36.2003

Zhang, W., Wen, H., Li, J., Lin, R., and Mcmanus, D. P. (2012). Immunology and immunodiagnosis of cystic echinococcosis: an update. Clin. Dev. Immunol. 2012:101895. doi: 10.1155/2012/101895
Zhang, W., You, H., Zhang, Z., Turson, G., Hasyet, A., and Mcmanus, D. P. (2001). Further studies on an intermediate host murine model showing that a primary Echinococcus granulosus infection is protective against subsequent oncospheral challenge. Parasitol. Int. 50, 279-283. doi: 10.1016/S1383-5769(01)00086-1

Zhang, W. B., Jones, M. K., Li, J., and Mcmanus, D. P. (2005). Echinococcus granulosus: pre-culture of protoscoleces in vitro significantly increases development and viability of secondary hydatid cysts in mice. Exp. Parasitol. 110, 88-90. doi: 10.1016/j.exppara.2005.02.003

Zoetendal, E. G., Raes, J., Van Den Bogert, B., Arumugam, M., Booijink, C. C., Troost, F. J., et al. (2012). The human small intestinal microbiota is driven by rapid uptake and conversion of simple carbohydrates. ISME J. 6, 1415-1426. doi: $10.1038 /$ ismej.2011.212

Conflict of Interest Statement: The authors declare that the research was conducted in the absence of any commercial or financial relationships that could be construed as a potential conflict of interest.

Copyright (c) 2018 Bao, Zheng, Wang, Zheng, He, Qi, Wang, Guo, Guo, Zhang, Zhang, Li and McManus. This is an open-access article distributed under the terms of the Creative Commons Attribution License (CC BY). The use, distribution or reproduction in other forums is permitted, provided the original author(s) and the copyright owner(s) are credited and that the original publication in this journal is cited, in accordance with accepted academic practice. No use, distribution or reproduction is permitted which does not comply with these terms. 